Linguistique, littérature, didactique

\title{
Les vieilles questions. Lire Fin de partie au lycée
}

Jean-Luc Picard

\section{(2) OpenEdition}

\section{Journals}

\section{Édition électronique}

URL : http://journals.openedition.org/pratiques/1886

DOI : 10.4000/pratiques.1886

ISSN : 2425-2042

\section{Éditeur}

Centre de recherche sur les médiations (CREM)

\section{Édition imprimée}

Date de publication : 15 décembre 2011

Pagination : 227-246

\section{Référence électronique}

Jean-Luc Picard, «Les vieilles questions. Lire Fin de partie au lycée », Pratiques [En ligne], 151-152 I

2011, mis en ligne le 16 juin 2014, consulté le 14 novembre 2019. URL : http://

journals.openedition.org/pratiques/1886 ; DOI : 10.4000/pratiques.1886

(C) Tous droits réservés 


\title{
Les vieilles questions. Lire Fin de partie au lycée
}

\author{
Jean-Luc Picard
}

"Lasciate ogne speranza, voi ch'intrate»

Dante, La divine comédie

\section{Fin de partie au programme de terminale}

C'est la deuxième année que l'œuvre de Beckett, Fin de partie ${ }^{(1)}$, est au programme de la classe de terminale littéraire (TL) ${ }^{(2)}$. Cette pièce, réputée difficile, a dû inquiéter de nombreux professeurs. Mais, heureuse surprise, la pièce n'a pas été rejetée par des élèves, plus déroutés par d'autres œuvres au programme comme les Pensées de Pascal ou les Mémoires de guerre du Général de Gaulle. Cet article fait la synthèse de deux ans d'étude du texte de Beckett en classe de terminale, la première année dans un grand lycée de Nîmes (Lycée Alphonse Daudet), et la seconde dans un lycée excentré de Tahiti (Lycée de Taravao). Des élèves bien différents donc, mais également intrigués, et parfois fascinés, par cette pièce de théâtre qui bouscule leurs habitudes de lecture et l'idée qu'ils se font du théâtre.

Cet article n'est pas à proprement parler un compte rendu, chronologiquement ordonné et didactiquement articulé, d'une séquence d'enseignement. Il essaie seulement de revenir sur un travail mené pendant deux ans. Les considérations qui vont suivre doivent beaucoup aux élèves, à leurs questions, à leurs incompréhensions parfois, mais aussi à leur fraîcheur, dispositions qui ont poussé l'enseignant à aller plus loin, à remettre en question ses hypothèses et ses certitudes. Le texte qui suit a pour seule prétention d'ouvrir quelques perspectives nouvelles dont il conviendra de mesurer l'intérêt didactique et la pertinence scientifique.

Dans les manuels scolaires, Fin de partie, pièce écrite en français par Beckett et

(1) Notre édition de référence est : Samuel Beckett, Fin de partie, Les Editions de Minuit, Paris, 1957.

(2) «D. Domaine : Littérature contemporaine - Euvres contemporaines françaises ou de langue française. Euvre : «Fin de partie » de Samuel Beckett» B.O, «Programme de littérature de la classe terminale de la série littéraire pour l'année scolaire 2010-2011 », Note de service n 2009-200 du 21-122009. 
créée le $1^{\circ}$ avril 1957 dans un théâtre de Londres, est classée dans la rubrique «théâtre de l'absurde » aux côtés de En attendant Godot et de quelques autres pièces de Ionesco. Ce classement rapide permet de s'accommoder de l'étrangeté ou de l'incongruité du texte. Pourtant Beckett, comme le fit Flaubert en son temps pour le « réalisme », s'est toujours insurgé contre son embrigadement dans un mouvement littéraire, contre un «absurde» dont sa pièce serait prétendument l'illustration ${ }^{(3)}$. L'importante biographie que James Knowlson a consacrée à notre auteur fait état de son irritation quand on tentait de définir ainsi son travail ${ }^{(4)}$.

Alain Badiou raconte, de son côté, de quelle manière, dans sa jeunesse, il a abordé Beckett : «... je ne pouvais voir en Beckett que ce que tout le monde y voyait. Un écrivain de l'absurde, du désespoir, du ciel vide, de l'incommunicabilité et de l'éternelle solitude, un existentialiste en somme. $"{ }^{(5)}$ Le confinement des personnages dans une pièce grise ne pouvait que rappeler une pièce antérieure, Huis-Clos de Sartre ${ }^{(6)}$.

Les lectures, «absurdes » ou « existentialistes », font peut-être bon marché de l'originalité de l'œuvre de Beckett. On ne peut cependant pas non plus se contenter, en classe, d'une simple contextualisation du texte. Contextualisation souvent abusive qui expliquerait, par exemple, le dénuement des personnages comme une référence aux camps de la mort. Certains critiques font même de la pièce grise où se déroule l'action une sorte d'abri antiatomique... Ces interprétations ne sont, bien sûr, pas à rejeter. Elles ont l'avantage de rassurer les élèves qui les retrouvent dans les manuels ou sur Internet, mais on peut avoir l'ambition de montrer qu' aucune interprétation n'épuise le sens d'un texte. Les élèves ne sont pas a priori tentés par une lecture nouvelle de l'œuvre, mais ils y souscrivent volontiers s'ils y trouvent de l'intérêt ou du plaisir. Ils sont d'ordinaire intéressés quand ils se rendent compte que ce qui leur paraissait seulement absurde, ou simplement farcesque, à la première lecture, est en réalité plus cohérent, et plus profond, qu'ils ne l'imaginaient.

Nous avons donc pensé qu'une lecture d'inspiration ethnocritique pouvait les stimuler parce qu'elle faisait apparaître des cohérences nouvelles et qu'elle était susceptible de les amener à s'interroger sur quelques grandes questions anthropologiques qui hantent depuis toujours l'humanité. De «vieilles questions » qui ne peuvent laisser insensibles des jeunes gens ou des jeunes filles qui abordent la philosophie en classe de terminale.

«Hamm - J'aime les vieilles questions. (Avec élan.) Ah les vieilles questions, les vieilles réponses, il n’y a que ça !» (p. 52)

Ces « vieilles questions » vont justifier une grande part de notre lecture attentive à la culture du texte, et partant, à la singularité de l'œuvre.

(3) «C'est pourquoi je n'ai jamais été d'accord avec cette notion de théâtre de l'absurde. Car il y a là un jugement de valeur. On ne peut même pas parler du vrai. », Charles Juliet, Rencontres avec Beckett, P.O.L, Paris, 1999, pp. 35-36. On reconnaît dans ce refus de tout jugement un écho de l'inquiétude de Hamm dans Fin de partie : «On n'est pas en train de... de... signifier quelque chose ? Et plus loin : «Une intelligence, revenue sur terre, ne serait-elle pas tentée de se faire des idées, à force de nous observer? (Prenant la voix de l'intelligence.) Ah, bon, je vois ce que c'est, oui, je vois ce qu'ils font! »,op. cit., p. 47.

(4) James Knowlson, Beckett, Actes Sud, Arles, 1999.

(5) Alain Badiou, Beckett. L'increvable désir, Pluriel, Hachette Littératures, Paris, 1995, p. 6.

(6) La pièce de Sartre a été mise en scène en 1944. 


\section{La polyphonie culturelle de l'œuvre}

Paradoxalement, il faut d'abord commencer par rendre le texte opaque, plus complexe pour faire découvrir aux élèves la polyphonie culturelle de l'œuvre, et se risquer à faire, avec eux, quelques hypothèses. A l'enseignant de donner l'exemple. Il semble assez aisé de montrer que la pièce de Beckett, faite de dialogues apparemment pauvres et répétitifs, fourmille en réalité d'allusions savantes et de citations cachées. Comment ne pas reconnaître, par exemple, dans le personnage de Hamm, cet aveugle sur un trône ridicule, l'évocation de cet autre roi aveugle que fut Edipe $^{(7)}$ ? Hamm est à la fois un roi déchu et une triste figure de carnaval au masque rouge sang. Peut-être une réminiscence du personnage du Masque de la Mort rouge de Poe. Hamm l'infirme annonce certainement le Bérenger du Roi se meurt de Ionesco que connaissent quelques élèves.

Les références bibliques sont légion dans la pièce. Les élèves polynésiens, qui connaissent mieux la Bible que leurs camarades métropolitains, se sont révélés des lecteurs perspicaces. Hamm évoque, par exemple, le «Mané, mané » (p. 24) du festin de Balthazar (Livre de Daniel, V). A la fin de la représentation, il appelle son père deux fois comme le fit le Christ en croix (p. 90). Ils ont donc été naturellement plus sensibles à ce que le texte a de blasphématoire ou de parodique : «Le salaud! Il n'existe pas » (p. 74) ou «Léchez-vous, les uns les autres!» (p. 89). Beckett sollicite aussi d'autres auteurs. Shakespeare, par exemple : «Mon royaume pour un boueux » (p. 36), s'écrie Hamm, parodiant Richard III, un autre roi déchu. Et enfin Baudelaire que Hamm tient à citer avec exactitude dans son monologue final :

« Tu appelais - (Un temps. Il se corrige.) Tu RECLAMAIS le soir; il vient-(Un temps. Il se corrige.) IL DESCEND : le voici. (Un temps.) Joli ça. » (p. 109)

On peut profiter de ce décryptage rapide pour faire constater que le thème de la lumière qui meurt, évoqué par un aveugle, semble important dans la pièce et qu'il est lié d'une certaine façon à celui de la royauté déchue. Peut-être faut-il rappeler Pascal qui fut aussi au programme de TL. La découverte de la densité culturelle du texte pique la curiosité des élèves et les invite à approfondir, à trouver un sens nouveau à ce qui ne leur paraissait qu'absurde.

Lors d'une première lecture faite à haute voix en classe, tous les élèves, métropolitains ou tahitiens, ont été très sensibles au comique farcesque de Beckett. Cette pièce qui met aux prises un aveugle et un boiteux appartient d'évidence à une tradition théâtrale ancienne. Il s'agit d'un exemplum ${ }^{(8)}$ moderne. Les farces et les contes du Moyen Age aimaient mettre en scène ce duo calamiteux et si peu chrétien.

Ce décryptage, un peu savant, a donc le double avantage de situer le texte dans l'histoire d'un genre et de montrer aux élèves de quelle manière se construit une œuvre. L'écriture hachée, discontinue, allusive de Beckett paraît faite de lambeaux arrachés à une culture ancienne, lointaine, apparemment dérisoire, mais essentielle pour en comprendre la construction.

(7) Voir Jean-Pierre Vernant : http://www.fabriquedesens.net/OEdipe-par-Jean-Pierre-Vernant

(8) Une Moralité de l'aveugle et du boiteux fut composée, par exemple, par André de la Vigne en 1496 pour la fête patronale de Seurre sur la commande de Philippe de Hochberg, gendre du duc Philippe II de Savoie et seigneur de Seurre. La pièce est conçue par l'auteur comme un épilogue pour le Mystère de saint Martin qu'il a écrit pour la même fête : elle met en scène deux infirmes confrontés au miracle possible de la guérison par l'intermédiaire des reliques de saint Martin : le boiteux refuse ce miracle, préférant continuer à gagner, en mendiant, assez d'argent sans travailler, et, guéri malgré lui, il injurie le saint ; l'aveugle guéri se réjouit et loue Dieu. Sur l'exemplum : Jean-Thiébaut Welter, L'exemplum dans la littérature religieuse et didactique $d u$ Moyen Âge : http://www.persee.fr/web/revues/home/prescript/article/rhef_0300-9505_1928_num_14_63_2468_t1_0218_0000_2 
Après avoir lu le texte à haute voix et avoir repéré les références culturelles, il n'est pas sans intérêt de s'interroger enfin sur le titre de l'œuvre : Fin de partie. Le mot «partie » est suffisamment polysémique pour donner lieu à de nombreuses hypothèses de lecture. La partie est un jeu, soit, mais c'est aussi ce qui divise le tout. La pièce serait donc un jeu, une réflexion sur la manière de finir de jouer. Une réflexion sur le théâtre, peut-être, et sur la vie, bien sûr... On connaît aussi l'intérêt de Beckett pour les échecs. Les fins de parties posent des problèmes qu'aiment résoudre les amateurs : les blancs jouent et gagnent en x coups. Hamm ne serait-il pas une sorte de roi d'échecs ? Une pièce du jeu importante certes, mais si peu mobile, si dépendante des autres pions. Et que font sur l'échiquier les poubelles de Nagg et Nell ? Des pièces mises de côté, dont on ne saurait que faire? Certains élèves voient en Clov, ce personnage à la démarche raide, une sorte de fou condamné à ne se déplacer que de biais. La pièce serait donc aussi une partie qui ne pourrait se terminer. Aux échecs, on parle de «pat» quand la fin est impossible. Mais, plus philosophiquement, la fin de la partie, c'est aussi l'accession à la totalité, le moment où le tout devient plus important que la somme des parties qui le constituent, l'instant où l'on accède enfin au sens. Nous y reviendrons.

Enfin il faut ramener les élèves à ces « vieilles questions » dont parle Hamm. Les personnages de Beckett ne cessent de s'interroger, souvent plaisamment, sur ce qui inquiète l'humanité. Comment passer de la vie à la mort? Quelles relations faut-il entretenir avec les anciens, les disparus? La lumière du soleil finira-t-elle par s'éteindre ? La Nature peut-elle cesser de se renouveler? Pourquoi procréer puisque tout est voué à disparaître ? C'est sur ces questions que nous avons choisi d'attirer l'attention des élèves parce qu'elles nous semblent essentielles à la compréhension profonde de l'œuvre et qu'elles en renouvellent l'approche.

Fin de partie, comme son titre l'indique, est une eschatologie. Finir la partie, c'est « finir de perdre » comme le dit Hamm (p. 108). Comment fait-on pour « passer » définitivement ? Comment franchir ce dernier seuil invisible ? Comment sauter définitivement le pas et accéder au non-être ou à quelque chose de qualitativement différent? Le drame des personnages de la pièce est qu'ils semblent ne pas pouvoir quitter la situation dans laquelle ils semblent englués. Hamm, plus solitaire à la fin de la pièce qu'au début, sait qu'il va devoir continuer à « jouer» seul, se demandant s'il est condamné à vivre éternellement cette suite infinie d'instants banals. S'il a bien, comme son fils et complice Clov, le sentiment que quelque chose est en cours, il ne sait quoi ni comment passer à autre chose. Mais ce « passage » dépend-il vraiment de lui ? Sa déception finale contredit l'espoir exprimé dès le début de la pièce : «... et un jour soudain, c'est un tas, un petit tas, l'impossible tas. » (p. 14). Vieille question : comment passe-t-on « soudain » de la somme des parties au tout que forme une vie ? Les hommes ont imaginé des rites pour donner un sens à ce qu'ils vivent, des cérémonies pour marquer les étapes de leur existence, mais ici, c'est d'un passage autrement plus important qu'il est question, puisque c'est celui de la vie à la mort, d'une suite d'instants vécus à une totalité dont on se demande si elle peut faire sens. Instant fugitif du passage, moment « absurde » qui défie la raison et dont l'œuvre de Beckett raconte l'attente improbable.

\section{Ce que c'est que passer}

Les élèves aiment donc lire à haute voix cette farce sinistre avec ses répliques étranges et méchantes. Mais on sent bien vite qu'au-delà de l'amusement quelque chose les touche, les " point», comme dirait Barthes. Que se passe-t-il donc vraiment sur scène qui peut les inquiéter, nous inquiéter ? ${ }^{(9)}$ Les personnages semblent l'ignorer. Quelque

(9) Nous avions à notre disposition, sur DVD, deux mises en scène, l'une du Théâtre des 13 Vents de Montpellier, et l'autre de Charles Berling, Collection COPAT, 2008. 
chose « se passe », répètent-ils, mais quoi ? Nous avons l'impression d'assister à une expérience incongrue, à une cérémonie dont on comprend mal l'utilité. Beckett explore, à partir de presque rien, avec un matériel scénique et verbal réduit, ce pur moment d'attente qui semble seul l'intéresser. Mais c'est ainsi qu'il nous tient, parce que cette attente désespérée, c'est la nôtre. Nous sommes plongés, avec les personnages, dans cette expérience curieuse et rare qui consiste à nous demander ce que nous sommes en train de vivre au moment où nous le vivons. Théâtre expérimental, dit Badiou. Alors que se passe-t-il vraiment dans Fin de partie? La pièce n'aurait-elle pour but que de nous faire réfléchir à ce que c'est que « passer»? Y aurait-il des rites de passage efficaces ? Les personnages espèrent en finir, disent-ils. Le passage est souhaité, soit. Mais quand aura-t-il lieu ? Qui peut les conduire de l'autre côté ? Et comment? On sent bien qu'ils ne peuvent plus supporter leur présent, morcelé, lacunaire et douloureux. Ils ne s'aiment plus, ils en ont assez de vivre ensemble et de se faire souffrir. Il leur faut vraiment « passer » à autre chose. Mais " passer », c'est aussi souffrir, c'est le sens premier du mot «passion». «Passer», c'est aussi faire un pas, nous dit le dictionnaire. Les personnages ont visiblement besoin, comme nous, lecteurs ou spectateurs, de faire un pas de plus pour saisir enfin le sens de ce qui se passe. Et ce pas ne sera jamais fait. C'est de cette attente dont nous parle Beckett, de ce moment où les instants disparates d'une vie, ces « instants nuls » (p. 109) devraient soudain faire sens.

Instants dont la vacuité est d'autant plus insupportable que Hamm, Clov, Nagg ou Nell semblent avoir eu un passé plus satisfaisant. Un passé blanc, comme le fond blanc du lac de Côme qui a tant marqué Nell lors de ses fiançailles alors que la lumière grisâtre qui baigne la scène paraît annoncer l'obscurité à venir, un noir à la fois espéré et redouté, signe d'une fin peu probable : «Fini, c'est fini, ça va finir, ça va peut-être finir. » (p. 13)

\section{Un personnage liminaire}

La pièce s'ouvre bizarrement sur une pantomime comique et maladroite de Clov. Une sorte de divertissement, clownesque et pascalien. Certainement des gestes mécaniques, routiniers pour tromper l'ennui. Mais peut-on parler de « gestes rituels »? La longue didascalie initiale rappelle les films burlesques qu'aimait Beckett, et particulièrement les comédies de Buster Keaton qu'il fit jouer plus tard dans le film qu'il tourna à New York $^{(10)}$. Si Clov a la « démarche raide et vacillante» (p. 11) des comiques du muet, il est pourtant le seul personnage mobile de la pièce. Tous les autres personnages dépendent de lui : Hamm est cloué dans son fauteuil d'infirme, au centre de la scène, et les parents sont enfermés dans leurs poubelles rangées sur un côté du plateau.

Clov, muet et affairé, semble avoir pour tâche première de manipuler des tissus. Il tire d'abord les rideaux des fenêtres, puis plie «soigneusement» et met sur son bras les "vieux " ${ }^{(1)}$ draps qui recouvrent les poubelles et Hamm. Beckett a trouvé une manière originale de lever «le torchon », comme on dit au théâtre. En effet, il n'y a pas de lever de rideau au début de la pièce, sinon ces draps que plie Clov et qui indiquent que quelque chose commence, que nous entrons dans la fiction de l'œuvre. Mais les élèves qui ont travaillé sur l'Odyssée, œuvre également au programme de TL, savent bien que le maniement des tissus est depuis longtemps réservé aux femmes : Calypso, Circé, Nausicaa,

(10) Film (1965), projeté en classe, montre un Buster Keaton vieillissant qui fuit sans cesse un regard inquisiteur, comme Hamm qui redoute le regard de Clov sur ses yeux. On se rend compte à la fin que l'œil qui effraie tant le personnage est son propre regard. Nous avons projeté en classe plusieurs extraits de comédies de Keaton, dont le personnage tragique et risible fascinait visiblement Beckett. Il eut même l'idée de le faire jouer dans En attendant Godot.

(11) Dusty dans la version anglaise de la pièce. 
Arété et Pénélope fabriquent ou offrent des tissus. L'épouse d'Ulysse ne tisse-t-elle pas, en attendant le retour de son mari, le linceul de son beau-père Laërte ? Ce rôle « féminin » échoit, dans la pièce de Beckett, à Clov qui plie, « avec soin », les draps. Clov - avatar d'Antigone ? - aide Hamm, aveugle, et roi déchu, à vivre : il veille sur son pipi et administre les médicaments. Mais c'est aussi ce même Clov qui doit aider son père adoptif à mourir $^{(12)}$. Il est de plus chargé de nourrir Nagg et Nell. Son domaine, c'est sa cuisine, située sur un côté du plateau : «Je m'en vais dans ma cuisine, trois mètres sur trois mètres, attendre qu'il me siffle. » (p. 14)

Dans cet univers étrangement privé de femmes, Clov assume le rôle féminin. Inversion des rôles propre au rite ? C'est à lui qu'incombe la charge réservée traditionnellement aux femmes, celle de nourrir et de faire passer. On ne peut plus compter sur Nell, la seule femme de la pièce. La mère de Hamm, prisonnière de sa poubelle, n'est d'ailleurs plus vraiment une femme : elle a perdu ses «guibolles» en faisant du tandem et elle se refuse énergiquement aux caresses et à la «bagatelle».

Clov paraît appartenir à ces personnages liminaires dont parle si justement Marie Scarpa ${ }^{(13)}$. Investi d'une mission particulière dans la réussite du passage qu'espère Hamm, il a d'évidence une fonction essentielle dans la séquence rituelle du passage telle que la décrit Van Gennep ${ }^{(14)}$. Clov est un personnage marginal à plusieurs titres. S'il boîte, il est pourtant le seul à pouvoir bouger. En outre, il paraît être aussi le seul à avoir quelque pouvoir dans cette «marge » qu'est le temps du spectacle. En manipulant les draps, il fait commencer le spectacle et c'est sur lui que compte Hamm pour le faire finir. Mais, étrangement, Clov révèle assez vite son incapacité à assurer le rôle rituel qui lui semble attribué. Il ne peut assumer complètement ses fonctions " marginales » et "féminines ». Son incapacité ontologique et rituelle est soulignée peut-être par la raideur de sa démarche. Sa claudication fait de lui un personnage à la fois comique et pathétique. Clov est un clown condamné à accomplir, sans conviction, la fonction rituelle qu'on attend de lui. S'il accomplit bien les gestes traditionnels qu'on faisait lors d'un décès, il ouvre les rideaux comme s'il voulait faciliter le départ de l'âme du mort, il a retourné le tableau, ou le miroir, qui se trouve au fond de la scène, pour que l'âme du défunt ne s'y perde pas, ses gestes sont apparemment sans effet. Hamm, son père adoptif, ne " passe » pas, pas plus que Nagg et Nell dans leurs poubelles. Hamm, à la fin de la pièce, doit gérer seul son passage. Clov ne peut lui fournir ni le cercueil, ni le drap qu'il lui réclame. Clov, lui-même, n'arrive pas d'ailleurs pas à « passer». Personnage liminaire, il reste figé sur le seuil de sa cuisine, en habits de voyage, prêt à partir mais « immobile jusqu'à la fin » (p. 108), incapable de quitter une pièce dans laquelle il est enfermé, et un père adoptif qui le martyrise. Figé à la porte, il ressemble à ces personnages de L'Ange exterminateur, un film de Buñuel (1962), qui, inexplicablement, ne peuvent plus franchir le seuil d'un salon. Hamm doute de pouvoir sortir un jour seul de ce lieu marginal dont il vérifie régulièrement, avec Clov, les limites :

«Hamm - Stop! (Clov arrête le fauteuil tout près du mur du fond. Hamm pose la main contre le mur. Un temps.) Vieux mur! (Un temps.) Au-delà c'est... l'autre enfer. (Un temps.) Plus près! Plus près! Tout contre!» (p. 39)

(12) Ces draps pourraient-ils être des linceuls ? L'hypothèse est à vérifier. Les élèves polynésiens savent que le travail sur l'étoffe végétale, le tapa, était aussi un travail réservé aux femmes, et que ces étoffes marquaient chaque passage important d'une vie. Particulièrement le tapa de naissance sur lequel on déposait le nouveau-né, et le tapa de mort dans lequel on enveloppait le défunt.

(13) Marie Scarpa, "Le personnage liminaire », Romantisme, ${ }^{\circ}$ 145, Armand Colin, pp. 25-35. Clov, comme la Teuse de Zola qu'étudie Marie Scarpa, fait basculer, en tirant les rideaux et en pliant les draps, les spectateurs dans la fiction théâtrale. Et comme elle, il boite.

(14) Arnold Van Gennep, Les Rites de passage, Picard, Paris, 1981 [1909]. Van Gennep distingue trois étapes dans la séquence rituelle : la séparation, la marge et l'agrégation. 
Beckett nous présente donc des personnages en détresse, englués dans une situation marginale, normalement provisoire, mais incapables d'accéder à l'agrégation finale que vise normalement le rite de passage. Personne ne peut rien pour eux. Ils flottent, le temps du spectacle, entre deux mondes ${ }^{(15)}$ et n'ont de cesse de s'interroger sur leur état. Nagg et Nell, restent eux aussi dans leurs poubelles, à demi enterrés, comme le sera plus tard Winnie dans Oh les beaux jours (1963). Ils ne sont guère vivants mais ils ne sont pas encore totalement " passés ». Ils font peut-être partie de ces morts qui n'arrivent pas vraiment à accéder à l'autre monde attendant une autre cérémonie qui leur assurera un passage définitif ${ }^{(16)}$ ? La pièce se termine sur un monologue de Hamm seul face au public. Tous attendaient beaucoup d'un passeur, d'un fils ou d'un père, qui ne peut malheureusement rien pour eux. Beckett insiste sur cet échec, revenant sans cesse sur une séquence rituelle, dont il n'ignore ni les codes ni la visée, mais dont il souligne sans cesse l'inefficacité.

«Hamm - Tu n'en as pas assez?

Clov - Si ! (Un temps). De quoi?

Hamm - De ce... de cette... chose.

Clov - Mais depuis toujours... (Un temps.) Toi non?

Hamm (morne) - Alors il n'y a pas de raison que ça change.

Clov - Ça peut finir. (Un temps.) Toute la vie les mêmes questions, les mêmes réponses.

Hamm - Prépare-moi. (Clov ne bouge pas.) Va chercher le drap. (Clov ne bouge pas.) » (p. 17)

Clov finit par renoncer, à la fin, à « préparer » Hamm et à lui donner le drap réclamé. Hamm devra se contenter de tendre, au moment où s'achève le spectacle, entre le public et lui, ce nouveau rideau, un peu ridicule, un mouchoir « taché de sang ». Mais ce geste est-il le geste rituel attendu ? Il semble en tout cas bien dérisoire et inefficace.

Les élèves s'interrogent avec raison sur ce mouchoir taché de sang qui recouvre le visage de Hamm et qui a, curieusement, assez peu inspiré les critiques ${ }^{(17)}$. Hamm tient à le manipuler lui-même. Est-il tapu, sacré ? Clov, chargé de manipuler d'autres tissus, n'a, semble-t-il, pas le droit de toucher à celui-ci. Hamm a même peur que Clov le soulève pendant son sommeil pour lui ôter ses lunettes noires. Ce linge protège ce qu'il a de plus intime, son regard, ce regard blanc d'aveugle que le spectateur ne verra d'ailleurs jamais. C'est Hamm, et pas Clov, qui se charge lui-même de plier «soigneusement» ce tissu qu'il «met délicatement dans la poche du haut de sa robe de chambre » (pp. 14-

(15) Arnold Van Gennep parle dans le chapitre premier qu'il consacre au « passage matériel » d'une zone entre deux territoires. Il note ceci à propos de la marge: «Quiconque passe de l'un à l'autre se trouve ainsi matériellement et magico-religieusement, pendant un temps plus ou moins long, dans une situation spéciale : il flotte entre deux mondes. C'est cette situation que je désigne du nom de marge, et l'un des objets du présent livre est de démontrer que cette marge idéale et matérielle à la fois se retrouve, plus ou moins prononcée, dans toutes les cérémonies qui accompagnent le passage d'une situation magico-religieuse ou sociale à une autre. ", Les Rites de passages, Editions Picard, 1981 [1909], p. 24.

(16) La littérature ethnologique recense de nombreux cas de doubles cérémonies funéraires : «Le cycle funéraire idéal était en effet marqué dans la plupart des sociétés polynésiennes par le déroulement successif de deux cérémonies (ou groupes de cérémonies) d'une importance symbolique cruciale. La première, qui avait lieu immédiatement après le décès, comportait un certain nombre de manipulations du corps du défunt (comprenant, à Tahiti notamment, l'embaumement, l'exposition et la dessiccation du cadavre). Elle se concluait (le plus souvent) par l'inhumation du corps. Plusieurs mois après se déroulait l'ultime cérémonie funéraire, lors de laquelle étaient prélevées les reliques du défunt, généralement à la suite de l'exhumation du cadavre dont la corruption était désormais achevée. " Alain Babadzan, Naissance d'une tradition. Changement culturel et syncrétisme religieux aux Iles Australes (Polynésie française), Collection Travaux et documents, $\mathrm{n}^{\circ} 154$, Editions de l'ORSTOM, Paris, 1982, p. 143.

(17) Une mise en scène présentée aux élèves présente même un mouchoir parfaitement blanc. 
15). Il ne le ressortira de cette poche qu'à la fin du spectacle pour le tendre à nouveau devant son visage. Comme tout linge, ce mouchoir, qui paraît particulièrement sacré, dissimule et révèle à la fois. Il cache le regard de celui qui le porte mais révèle un saignement étrange, une blessure secrète dont on ne sait rien. Une souffrance intime que le texte n'évoque qu'indirectement.

Aucun élève ne connaît, bien sûr, la légende de Véronique, la femme qui a essuyé le visage du Christ pendant la montée au Calvaire. Sur le voile de Véronique (ou Bérénice, « celle qui porte la victoire ») s'est imprimée la figure sanglante du Christ. Le voile de Véronique est non seulement la première icône chrétienne ${ }^{(18)}$ mais aussi une image « volée ». Mais le Christ, à la différence de Hamm, a accepté ce vol comme s'il était nécessaire à sa Passion, et à son « passage ». Le linge féminin lui a permis d'aller au bout de son calvaire, jusqu' au linceul du tombeau et à la Résurrection finale. En ne permettant pas à Clov de toucher au mouchoir qui lui couvre le visage, Hamm refuse « sa »Véronique ${ }^{(19)}$. En refusant que Clov touche à ce linge ${ }^{(20)}$, serait-il responsable de l'échec du rite ?

L'agrégation finale attendue n'a donc pas lieu. A la fin du spectacle, Hamm tend, aux spectateurs ce mouchoir qu'il qualifie familièrement de «vieux linge ", essayant ainsi de le désacraliser. Ce mouchoir tendu n'est plus qu'une sorte d'écran usé par la répétition d'un rite désormais inefficace. Hamm, qui ne peut plus compter que sur lui-même, ne se fait aucune illusion sur ce qui n'est plus, en réalité, qu'un jeu dérisoire et théâtral. $\mathrm{Sa}$ « passion » devient alors parodique et risible.

«Puisque ça se joue comme ça... (il déplie le mouchoir)... jouons ça comme ça... (il déplie)... et n'en parlons plus. (Il tient à bout de bras le mouchoir ouvert devant lui.) Vieux linge! (Un temps.) Toi - je te garde.

Un temps. Il approche le mouchoir de son visage. RIDEAU », p. 110.

Le mouchoir, linge essentiel et rituel du passage, redevient accessoire de théâtre, objet manipulé par un initié désenchanté. Le rite s'est dégradé en comédie. La Passion, séquence rituelle du passage s'il en est, n'est plus qu'un spectacle qui peut être rejoué à volonté. Le personnage principal s'est condamné lui-même à la solitude et à la répétition douloureuse de la même scène, comme un de ces damnés dont les Anciens et Dante avaient peuplé leur Enfer.

(18) Cette légende a certainement joué un rôle important aux premiers temps du christianisme contre l'iconoclastie et a inspiré, bien plus tard, les peintres, fascinés par cette mise en abyme, par l'idée de produire l'image de cette femme qui présente l'image divine. Voir par exemple Pontormo ou Memling au $X^{\mathrm{e}}$ siècle.

(19) Beckett n'ignorait pas qui était Véronique, ne serait-ce que parce qu'il connaissait l'œuvre de son compatriote Yeats. On peut lire ce poème de Yeats consacré à Véronique intitulé Veronica Napkin, où apparaît le tissu ensanglanté :

«THE Heavenly Circuit ; Berenice's Hair ;

Tent-pole of Eden; the tent's drapery;

Symbolical glory of the earth and air !

The Father and His angelic hierarchy

That made the magnitude and glory there

Stood in the circuit of a needle's eye.

Some found a different pole, and where it stood

A pattern on a napkin dipped in blood. "

William Butler Yeats (1865-1939)

http://ariel.synergiesprairies.ca/ariel/index.php/ariel/article/viewFile/479/471

(20) La vénération qui entoure le Saint Suaire de Turin (voir M. Pochon, « Le Linceul du Christ : preuve ou épreuve? »,Études 2001/4, Tome 394, p. 497-509) montre la force de l'image, le pouvoir qu'elle a sur l'imagination. Le suaire est non seulement une relique, puisque le sang et les humeurs du supplicié ont été « étanchées» par lui («Old stancher» dit Hamm dans la version anglaise de Fin de partie), mais aussi une reproduction, une icône, qui entend faire triompher, dans la guerre des images qui a sévi dans la Chrétienté, les adversaires des iconoclastes. 


\section{Une après-midi d'avril}

Si on ne sait exactement ce qui a conduit les personnages dans cette marge grisâtre dont ils ne peuvent sortir, on sait en revanche qu'ils eurent un passé qui leur paraît rétrospectivement heureux.

«Clov-Nous aussi on était jolis - autrefois. Il est rare qu'on soit pas jolis - autrefois. » (p. 59)

Nell, la mère de Hamm, se souvient avec émotion de ses fiançailles d'avril au bord du lac de Côme. Cet autrefois est un Paradis dont les personnages ont été chassés. Ils ont tous de la difficulté à se détacher de ce passé mythique dont leur imagination entretient l'espoir du retour, rendant ainsi plus difficile l'acceptation d'une mort par ailleurs souhaitée. Pour Nagg et Nell, il y eut une vraie chute qui les fit sortir de cet Eden lointain. Leur accident de tandem leur coûta leurs « guibolles », ce qui explique leur immobilisation dans une poubelle et leur dépendance à l'égard de leur fils. Pour Hamm, on ne sait pas vraiment ce qui l'a conduit sur sa chaise roulante. On sait seulement qu'il regrette une jeunesse heureuse, différente de son présent grisâtre. Pour Clov, nous n'avons aucune information sur la cause de la raideur qui l'empêche de s'asseoir. Le temps passé, à les en croire, fut un temps coloré, et sexuellement actif. Très différent, en tout cas, du monde cendreux et marginal qui est le leur ${ }^{(21)}$.

«Nell - C'était sur le lac de Côme. (Un temps.) Tu peux le croire?

Nagg - Quoi?

Nell - Que nous nous sommes promenés sur les bords du lac de Côme. (Un temps.) Une après-midi d'avril.

Nagg - On s'était fiancés la veille.

Nell - Fiancés. » (pp. 33-34)

Les personnages attendent un dernier événement qui leur fera enfin quitter cet entredeux sinistre. Mais il semble que l'écoulement du temps se soit subitement ralenti. Le temps lui-même hésite et balbutie. La didascalie «Un temps. » qui troue régulièrement le texte de Beckett vient régulièrement le rappeler au lecteur de la pièce. Les personnages espèrent que quelque chose suit son cours, et que la fin arrivera fatalement.

L'allusion rapide au mois d'avril faite par Nell n'est pas sans intérêt. Si on est vraiment attentif au texte, ce mois, dont le souvenir vivace s'oppose au présent hivernal, froid et gris, de la marge, joue un rôle important dans la construction de l'œuvre et lui donne une partie de sa cohérence. L'ethnocritique a suffisamment insisté sur l'importance du calendrier pour que nous prenions en compte cette référence au mois printanier où la nature apparemment morte se réveille soudain. Hamm, malgré son envie d'en finir, ne peut lui-même s'empêcher de songer avec nostalgie à ce renouveau. Il a du mal à

(21) Voir, à propos des cendres, la pièce radiophonique de Beckett Cendres (Embers). Avant « La dernière bande », Beckett s'est lancé dans l'écriture d'une pièce radiophonique en anglais qu'il destine à Donald Mc Whinnie, metteur en scène, qu'il a laissée de côté : «Cendres ». Cette pièce, qu'il retravaille par la suite, sera adressée en 1959 à la BBC. Elle sera sélectionnée par le jury de la RAI pour le prix Italia. Le succès de Cendres conduira la $\mathrm{BBC}$ et la radio française à lui passer deux nouvelles commandes radiophoniques : ce sera Paroles et musique (musique de John Beckett) et Cascando, sur une musique de Mihalovici (diffusé en France en 1963). » En archives sur France Culture : http://www.franceculture.com/emission-fictions-perspectives-contemporaines-cendres-desamuel-beckett-2010-09-25.html Ce thème de la cendre est évidemment à approfondir si on se livre à une analyse plus fine de la pièce qui reprend, en partie, la liturgie pascale. Le Mercredi des Cendres suit les Rameaux (Pâques fleuries) et précède le repas de la Cène, et la Crucifixion (voile de Véronique et appel au père). 
abandonner tout espoir de retour. Il se prend à souhaiter une répétition qui annulerait définitivement toute fin de partie.

«Hamm - Mais derrière la montagne ? Hein ? Si c’était encore vert? Hein ? Flore ! Pomone! (Un temps.) Cérès! Tu n'aurais pas besoin d'aller plus loin. » (p. 54)

Plus loin, il espère encore : «C'est une feuille ? Une fleur ? », p. 101.

L'allusion aux fêtes de Pomone et de Flore n'est pas seulement le signe d'une emphase ridicule et déplacée, une référence ironique et absurde à une culture inutile. Elle exprime la vieille inquiétude que ressentent les hommes quand la Nature paraît mourir. La grisaille, le froid ne sont-ils qu'un sommeil, ou l'annonce d'une mort réelle ? Les fêtes de Flore, de Pomone, et plus largement tous les rites qui accompagnaient le culte de Cérès, célébraient la fin de cette peur et la joie éprouvée lors du renouveau printanier. Le mois d'avril montre qu'il n'est pas vain d'espérer malgré la mort apparente. Ces fêtes du renouveau célébraient aussi le mystère d'une « mort» nécessaire pour qu'il y ait renouveau et résurrection ${ }^{(22)}$. Le mois d'avril dont rêvent ensemble Nell et Hamm est donc non seulement le souvenir heureux d'une période lointaine mais la promesse d'un renouveau possible qui vient contrarier leur désir d'en finir. Hamm raconte à Clov l'histoire de ce fou qui croyait que le monde n'était que cendres :

«Il faisait de la peinture. Je l'aimais bien. J'allais le voir, à l'asile. Je le prenais par la main et le traînais devant la fenêtre. Mais regarde! Là, tout ce blé qui lève ! Les voiles des sardiniers! Toute cette beauté ! (Un temps.) Il m'arrachait sa main et retournait dans son coin. Epouvanté il n'avait vu que des cendres. » (pp. 60-61)

Hamm est-il devenu, à son tour, ce fou qui croit que tout est définitivement cendres? On sent bien qu'il n'arrive pourtant pas à se résoudre à abandonner tout espoir. "Lasciate ogne speranza, voi ch'intrate » lit-on dans La Divine Comédie. Il demande souvent à Clov de prendre sa lunette et de lui confirmer que le monde est bien gris cendre. Mais on sent aussi qu'il attend encore quelque chose : " On est quel mois ? (p. 86). Non, on n'est plus en avril. Il fait froid, il gèle et Clov lui confirme que tout est vraiment «mortibus ».

Il faut profiter du goût des élèves pour la mythologie. L'histoire de Cérès-Déméter ${ }^{(23)}$, dont Hamm cite le nom, les intéresse et leur montre sur quel substrat ancien se construit le texte. Mais le mythe est aussi mystère. Il rappelle qu'il faut accepter de passer sous la terre pour pouvoir renaître. Le culte de Déméter ritualisait ce passage mystérieux de la mort à la vie. Les mystères d'Eleusis initiaient les fidèles à cette énigme du renouveau printanier et de la Résurrection possible après la mort. Les rites pratiqués à l'occasion de ces fêtes concernaient au premier chef les céréales et particulièrement le blé. Avril était le mois où les graines qu' on avait longtemps cru mortes germaient à nouveau. La semence, sperma en grec, qui concentre toute la charge symbolique du mythe, fascine d'abord parce qu'elle renferme en elle le principe de son développement, mais elle inquiète aussi car elle enseigne qu'il faut accepter de mourir pour renaître. L'évangile de Jean reprendra, plus tard, le mythe pour en tirer sa morale chrétienne : «Si le grain de blé qui est tombé en terre ne meurt, il reste seul ; mais, s'il meurt, il porte beau-

(22) Ovide, dans les Métamorphoses (XIV), raconte l'histoire de Pomone, la déesse des jardins des Romains, représentée avec des fruits et un rameau à la main. Courtisée par Vertumne, déguisée en vieille femme, la belle déesse finit par céder à son amant. La nymphe Flore, dont parle aussi Hamm, et dont le nom grec, Cloris, évoque le vert du printemps, est liée, elle, plus particulièrement aux fleurs. En son honneur, les Jeux floraux étaient célébrés à la fin avril.

(23) Déméter : étymologiquement, la Terre-mère. 
coup de fruits. Celui qui aime sa vie la perdra, et celui qui hait sa vie dans ce monde la conservera pour la vie éternelle. » (Jean, 12, 24-25).

Comment ne pas remarquer que cette référence aux fêtes céréalières d'avril rappelle ce qui nous est dit, dès le début de la pièce, à propos de la germination ? La question de Hamm qui semblait « absurde » est finalement très cohérente :

« Hamm - Tes graines ont levé ?

Clov - Non.

Hamm - Tu as gratté un peu voir si elles ont germé.

Clov - Elles n'ont pas germé.

Hamm - C'est peut-être encore trop tôt.

Clov - Si elles devaient germer elles auraient germé. Elles ne germeront jamais. » (p. 26)

Hamm, qui veut en finir, attend pourtant que les graines germent. Mais il sait aussi qu'il faut abandonner tout espoir de retour éternel. Il se moque de cette attente dans le roman qu'il compose péniblement :

« Mais enfin quel est votre espoir? Que la terre renaisse au printemps ?» (p. 71)

Cette contradiction définit le personnage. Qu'il lui est difficile de renoncer au retour printanier!

«Assez, il est temps que cela finisse, dans le refuge, aussi. (Un temps.) Et cependant, j'hésite, j'hésite... à finir. Oui, c'est bien ça, il est temps que cela finisse et cependant j'hésite encore à - (bâillements) - à finir. » (p. 15)

Les enfants de Provence, région où Beckett résida quelque temps à la fin de la guerre $^{(24)}$, connaissent cette tradition qui consiste à mettre, dans une coupelle, le 4 décembre, jour de la Sainte Barbe, sur un peu de coton humide, des grains de blé pour les faire germer. Trois semaines plus tard, à Noël, en plein solstice d'hiver, quand la nuit semble définitivement s'installer sur la terre, les jeunes pousses vertes annoncent que la Nature, malgré les apparences, est toujours active, et qu'il y aura bien un mois d'avril, un jour de résurrection.

Beckett va peut-être encore plus loin dans l'exploitation de la symbolique céréalière. Pourquoi Hamm refuse-t-il la bouillie à son père ? Refuse-t-il simplement la solidarité intergénérationnelle ou, plus radicalement, l'offrande céréalière qu'on faisait traditionnellement aux morts afin de les aider à passer vraiment? Quand Nagg sort la tête de sa poubelle, et réclame plusieurs fois à manger : «Ma bouillie !» (p. 21), Hamm se révolte contre cette obligation et s'écrie alors :

« Ah il n’y a plus de vieux! Bouffer, bouffer, ils ne pensent qu'à ça!», p. 21.

Finalement il est incapable de donner à son père la bouillie réclamée. C'est le même refus que lui opposera Clov, son propre fils, quand il lui réclame un drap et un cercueil pour en finir :

« Tu n'auras plus jamais de bouillie.» (p. 21).

Nagg, ce père à demi enterré dans sa poubelle, devra se contenter d'un biscuit. Plus tard quand Hamm raconte son roman à son père, il fait état, pour le narguer, de ce blé

(24) Il se réfugia à Roussillon dans le Vaucluse. 
qu'on trouve encore en abondance et dont on peut faire de la bouillie pour nourrir ses enfants :

« Du blé, j’en ai, il est vrai dans mes greniers. Mais réfléchissez, réfléchissez. Je vous donne du blé, un kilo, un kilo et demi, vous le rapportez à votre enfant et vous lui en faites - s'il vit encore - une bonne bouillie (Nagg réagit), une bonne bouillie et demie. Bien nourrissante. ${ }^{(25)}$ (p. 71)

Le père nourri devient l'enfant de son fils. Juste retour des choses que signifiait le rite céréalier. Hamm sent bien que ce père est en quelque sorte un mort en souffrance, mais il lui refuse, par vengeance, l'offrande rituelle due ${ }^{(26)}$. Nagg ne «passera » pas. Il questionne ainsi Clov qui vient de rabattre le couvercle de la poubelle :

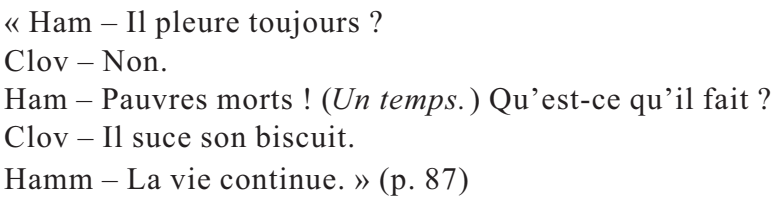

Hamm veut pourtant se débarrasser de tous ces morts qui l'entourent et le pressent. Il veut en finir avec cette charité imposée, avec les devoirs rituels dus aux disparus. Dans la Polynésie ancienne, ces morts ainsi repoussés n'auraient eu aucune chance de devenir des ancêtres, des tupuna. Hamm est celui qui veut rompre avec le rite, quitte à blasphémer :

« Tous ceux que j'aurais pu aider. (Un temps.) Aider! (Un temps.) Sauver! (Un temps.) Ils sortaient de tous les coins ${ }^{(27)}$. (Un temps.) Mais réfléchissez, réfléchissez, vous êtes sur terre, c'est sans remède! (Un temps.) Allez-vous en et aimez-vous ! Léchez-vous les uns les autres! (Un temps. Plus calme.) Quand ce n'était pas du pain c'était du mille-feuille. (Un temps. Avec violence.) Foutez le camp, retournez à vos partouzes!» p. 89.

Hamm semble vouloir en finir avec le rite, avec l'offrande rituelle céréalière, avec la symbolique chrétienne du pain qu' on partage, et peut-être avec ce pain qui devient le corps du Christ. Personne ne reviendra sous terre : les morts sont condamnés à rester " sur terre », " c'est sans remède ». La violence de la réplique révèle certainement une souffrance. Le personnage jusqu'à la fin de la pièce lutte contre lui-même et contre tout espoir rituel. Il repousse la symbolique de la graine. Mais il veut en finir vraiment, seul, et sortir enfin de cette marge où tout est répétition, douleur et comédie. Crucifié sur son fauteuil, il sait qu'il n'a aucune aide à attendre d'un Père compatissant. Pourquoi seraitil, lui, compatissant?

(25) Plus loin, Hamm parle de la manne que Dieu donna aux Hébreux pour les nourrir, p. 71

(26) L'offrande céréalière faite traditionnellement aux morts imposait une solidarité réelle avec les disparus. Toutes les sociétés ont essayé de régler, par des rites divers, les relations entre vivants et morts. Les morts récents posaient particulièrement problème. Les élèves polynésiens savent à quel point les mauvais morts peuvent être parfois dangereux pour les vivants : on impute encore souvent en Polynésie les malheurs d'une famille à un mort que parfois on déterre pour le brûler. En Europe centrale, on déposait dans les tombes des pains de formes diverses. On pouvait aussi apporter des bouillies. On ne peut s'étonner que le blé, qui est la céréale du passage, fasse partie du rituel. Voir Marianne Mesnil et Assia Popova, «L'offrande céréalière dans les rituels funéraires du sud-est européen », http://civilisations.revues.org/index1412.html.

(27) Comment ne pas penser au voyage d'Ulysse chez les Cimmériens ? 


\section{L'Office des Ténèbres}

La pièce de Beckett est une pièce de grisaille et de cendres. Cette œuvre crépusculaire pose, de manière récurrente, la question de la fin de la lumière. Hamm attend le passage de la lumière à l'obscurité. Le gris qui est la couleur de la marge est signe d'une lente dégradation dont le noir absolu est la limite ${ }^{(28)}$. Si les graines ne germent plus et que la lumière diminue, c'est que la fin de partie est proche, que nous arrivons au seuil attendu.

« Clov - Je vois ma lumière qui meurt.

Hamm - Ta lumière qui - ! Qu'est-ce qu'il faut entendre ! Eh bien, elle mourra tout aussi bien ici, ta lumière. Regarde-moi un peu et tu m'en diras des nouvelles, de ta lumière. " (p. 25)

Et plus loin, Hamm, l'aveugle aux yeux blancs, prédit à Clov :

«Un jour tu seras aveugle. Comme moi. Tu seras assis quelque part, petit plein perdu dans le vide, pour toujours, dans le noir. » (p. 51)

Mais Hamm, toujours aussi contradictoire, aime aller sous la fenêtre, avide des dernières lueurs : «Je veux sentir la lumière sur mon visage. » (p. 83)

La fin de sa lumière n'a pas encore, pour Hamm, signifié la fin de la partie. Il a du mal à renoncer au soleil :

«Hamm - Et le soleil?

Clov (regardant toujours) - Néant.

Hamm - Il devrait être en train de se coucher pourtant. Cherche bien.

Clov (ayant cherché) - Je t'en fous.

Hamm - Il fait donc nuit déjà ?

Clov (regardant toujours) - Non.

Hamm - Alors quoi ?

Clov (de même) - Il fait gris. (Baissant la lunette et se tournant vers Hamm plus fort.)

Gris! (Un temps. Encore plus fort.) GRRIS !

Clov - Noir clair. Dans tout l'univers.

Hamm - Tu vas fort. (Un temps.) Ne reste pas là, tu me fais peur.

Clov - Pourquoi cette comédie tous les jours?

Hamm - La routine, on ne sait jamais. » (pp. 46-47)

La pièce est une Genèse à rebours. La séparation que Dieu crée entre la nuit et la lumière est peu à peu annulée. Tout redevient incertain. C'est le moment angoissant qui précède le passage du seuil, la régression vers un noir espéré et redouté à la fois. «Tu réclamais le soir, il descend...».

La pièce offre quelques variations, parfois comiques, sur la mort de la lumière. Les élèves sont sensibles à cet humour noir qui joue avec les peurs anciennes. On peut donner, comme exemple, ce quiproquo amusant lorsque Hamm demande des nouvelles de la mère Pegg, la voisine :

«Hamm - Il y a de la lumière chez la Mère Pegg?

Clov - De la lumière ! Comment veux-tu qu'il y ait de la lumière chez quelqu'un?

(28) La culture ancienne polynésienne opposait deux concepts, l'ao et le po, la lumière et l'obscurité. Le po était le monde des origines, celui des morts et des ancêtres. Et d'une certaine façon il désignait aussi l'univers féminin. Le monde humain, masculin, était celui de l'ao. Le christianisme imposé transforma cette opposition : le po fut assimilé à l'enfer, au mal. Voir Alain Babadzan, op. cit., pp. 124-151. 
Hamm - Alors elle s'est éteinte.

Clov - Mais bien sûr qu'elle s'est éteinte ! S'il n'y en a plus c'est qu'elle s'est éteinte.

Hamm - Non je veux dire la Mère Pegg.

Clov - Mais bien sûr qu'elle s'est éteinte ! Qu'est-ce que tu as aujourd'hui ?

Clov - Je suis mon cours. (Un temps.) On l'a enterrée ?», p. 58.

Beckett affectionne ces petits numéros à la fois clownesques et tragiques qui lient mort et obscurité. Clov reproche plus tard à Hamm d'avoir refusé de l'huile à la mère Pegg qui en avait besoin pour sa lampe ${ }^{(29)}$ : «Tu sais de quoi elle est morte, la Mère Pegg ? D’obscurité. » (p. 97).

Ces variations sur la lumière qui meurt nous amènent à parler de Pâques, la fête chrétienne de la lumière qui renaît, fête à laquelle le texte se réfère implicitement et explicitement. La liturgie de la semaine pascale reprend sous la forme linéaire d'un récit la vieille symbolique du renouveau d'avril. Pâques signifie bien "passage». La Pâque des Juifs rappelle la fuite d'Egypte, la libération d'un peuple asservi qui sacrifie un agneau avant de se mettre en route. La Pâques chrétienne, elle, syncrétise l'apport ancien, juif et grec, et propose une interprétation nouvelle. La fête céréalière grecque et la commémoration juive deviennent la fête de la Résurrection du Christ qui, par sa mort, sauve les hommes. Tous les éléments antérieurs sont réagencés et dramatisés au cours d'une semaine qui voit le triomphe de la lumière : le Mercredi Saint des Ténèbres ${ }^{(30)}$, le blé du dernier repas du Jeudi Saint, la mort du Vendredi Saint, et la Résurrection du Dimanche de Pâques. Si le grain ne meurt... C'est la mort vaincue, le passage mystérieux que fête la Chrétienté. Le sang du sacrifice explique-t-il les visages rougis de Clov et de Hamm, ainsi que le mouchoir sur le visage ? Fin de partie est d'évidence une Passion où ne triomphe aucune lumière. Hamm est un fils souffrant qui n'a pas trouvé la compassion d'une Véronique ou d'un père. Beckett multiplie les références aux Evangiles: Hamm, crucifié sur son fauteuil, doit aller seul vers la mort, mais il ne pourra s'empêcher d'implorer deux fois son père, comme le fit son modèle divin sur le Golgotha ${ }^{(31)}$ :

«Père! (Un temps. Plus fort.) Père ! (Un temps.) Bon. (Un temps.) On arrive. » (pp. 109-110)

Simple parodie, " chant d'à côté », jeu d'intellectuel, ou réflexion profonde sur la difficulté à passer? Quand, dans la première didascalie, Clov plie soigneusement les draps et ricane en découvrant Hamm et les poubelles occupées, on peut y voir la réécriture parodique du récit de la Résurrection de Jean. Mais ici il n'y a pas de Saintes Femmes ni d'apôtres étonnés par le tombeau vide et le suaire plié ${ }^{(32)}$. Au contraire, tout est prêt pour rejouer une nouvelle fois la partie.

(29) Il est difficile de ne pas penser aux lampes de l'histoire des vierges folles et des vierges sages de l'Evangile (Mathieu, 25).

(30) C'est ce mercredi, qui rappelle celui des Cendres au début du Carême, que débute, dans la liturgie catholique, la méditation sur la mort et les péchés des hommes. On célébrait les jours suivants l'Office des Ténèbres au cours duquel on éteignait une à une les bougies de l'autel, rituel inverse de celui de l'Avent.

(31) «Mon Dieu, mon Dieu, pourquoi m'as-tu abandonné ?» Marc, 15, 34. Cette parole du Christ est ellemême une citation du psaume 22 .

(32) Evangile de Jean, chapitre 20 : «[1] Le premier jour de la semaine, Marie de Magdala se rendit au sépulcre dès le matin, comme il faisait encore obscur ; et elle vit que la pierre était ôtée du sépulcre. [2] Elle courut vers Simon Pierre et vers l'autre disciple que Jésus aimait, et leur dit: Ils ont enlevé du sépulcre le Seigneur, et nous ne savons où ils l'ont mis. [3] Pierre et l'autre disciple sortirent, et allèrent au sépulcre. [4] Ils couraient tous deux ensemble. Mais l'autre disciple courut plus vite que Pierre, et arriva le premier au sépulcre ; [5] s'étant baissé, il vit les bandes qui étaient à terre, cependant il n'entra pas. [6] Simon Pierre, qui le suivait, arriva et entra dans le sépulcre ; il vit les bandes qui étaient à terre, [7] et le linge qu'on avait mis sur la tête de Jésus, non pas avec les bandes, mais plié dans un lieu à part. [8] Alors l'autre disciple, qui était arrivé le premier au sépulcre, entra aussi ; et il vit, et il crut. [9]" 
La fête de Pâques est encore évoquée, dans la pièce, dans la fameuse histoire du pantalon que Nagg raconte à Nell. L'histoire débute avant les fêtes de fin d'année. Un Anglais, qui a besoin d'un pantalon rayé pour le Nouvel An, va voir un tailleur. Mais ce tailleur ne réussit pas à confectionner à temps le vêtement. D'abord c'est le fond qui est raté, puis l'entrejambes, et enfin la braguette.

« Enfin bref, de faufil en aiguille, voici Pâques Fleuries et il loupe les boutonnières. »(p. 35)

L'histoire commence pendant la période de l'Avent, période où, dans certains pays, on allume chaque dimanche une bougie nouvelle, et où, dans d'autres, on met des grains de blé à germer. Et elle se termine donc à «Pâques Fleuries » ${ }^{(33)}$, pour l'équinoxe de printemps, le jour des Rameaux. Là encore, beaucoup de critiques n'ont pas relevé la pertinence de l'allusion de Beckett. " Pâques Fleuries » n'est pas simplement une expression surannée et vaguement ironique qui montrerait que Nagg raconte, comme il le dit, de plus en plus mal. En réalité, il semble bien que rien ne soit laissé au hasard dans la pièce. Lors de ce dimanche des Rameaux, on défilait, comme aux fêtes de Cérès, avec des rameaux en fleur, annonçant ainsi la victoire prochaine de la Vie sur la Mort. C'est ce dimanche, qui inaugure la Semaine Sainte et donne son sens de la liturgie pascale. Et c'est, bien sûr, ce dimanche de Pâques fleuries que choisit le tailleur pour « louper » les boutonnières du pantalon. Pas de boutons donc pour Pâques fleuries.... Encore une plaisanterie de Beckett. Les graines n'ont pas germé et les boutons ne sont plus là. La création du tailleur est un échec. Mais l'histoire du pantalon a une chute. L'Anglais fait remarquer au tailleur que Dieu a créé le monde en six jours et qu'il n'est pas capable, lui, de faire en quatre mois un pantalon. Le tailleur, qui tient en piètre estime la création divine, lui répond alors :

« Mais Milord! Milord! Regardez - (geste méprisant, avec dégoût) - le monde... (un temps) ... et regardez - (geste amoureux, avec orgueil) - mon PANTALON !» (pp. 35-36)

\section{Le sexe oublié}

Le monde créé par Dieu est certainement encore plus raté encore que le pantalon du tailleur. Toute création d'ailleurs est condamnée à l'échec, minée par la mort. Nagg se rend compte qu'il ne sait plus raconter son histoire, et Hamm, qui écrit un roman, est incapable d'en trouver la fin. Il se contente de coller des lambeaux de phrases, et de bricoler une parodie de récit de Noël. Et que raconte vraiment le récit de la Passion du Christ? L'histoire d'un fils que son père laisse crier et sacrifie. Il n'est donc pas donc étonnant que la pièce lie création et procréation. Hamm se méfie de tout ce qui pourrait inscrire l'humanité dans l'illusion de la durée, ce que le rite ou la religion tentent de faire symboliquement. Le sexe est accusé de perpétuer l'espèce. Hamm ne fait plus l'amour

(33) Cette expression qui désigne les Rameaux est-elle un souvenir des récits du Moyen Age, du Roman de Brut de Wace (XII ${ }^{\mathrm{e}}$ siècle) que connaît certainement le lettré qu'est Beckett ? Elle est encore utilisée en Normandie. Les élèves cultivés se souviennent peut-être de la rencontre, dans les Confessions, de Jean-Jacques avec Mme de Warrens qui a lieu aux Rameaux. "C'était le jour des Rameaux... ", écrit-il dans les Confessions, Livre II. Mais cette scène célèbre est à nouveau racontée dans les Rêveries d'un promeneur solitaire (1782) : «Aujourd'hui jour de Pâques fleuries, il y a précisément cinquante ans de ma première connaissance avec Mme de Warrens. » L'expression «Pâques Fleuries » convient pour parler de ce dimanche qui annonce le bonheur, parfait puisqu'il est champêtre : «Je ne désirais rien que la continuation d'un état aussi doux. » Une nouvelle naissance, pour l'orphelin qu'est Rousseau. Sur «Pâques fleuries », voir A. Van Gennep, Le Folklore français, I, Bouquins, Paris, 1998, pp. 972-1010. 
qu'en rêve. La graine, sperma, ne doit plus germer, contrairement à ce qu'il fait semblant d'attendre. Tout ce qui se rapporte à l'entrejambes est moqué. C'est bien l'entrejambes du pantalon, puis la braguette qui posent problème au tailleur. Et quand Clov fabrique un chien pour rassurer Hamm, il « oublie » curieusement le sexe :

« Hamm - Tu as oublié le sexe.

Clov (vexé) - Mais il n'est pas fini. Le sexe se met en dernier. » (p. 56)

Plus tard, Clov, avec sa lunette, croit apercevoir quelqu'un. Hamm est alors inquiet : il pensait, avec ses compagnons de marge, faire partie des derniers hommes. Et si l'histoire recommençait avec l'apparition d'un intrus capable de se reproduire ? La pièce grise ne doit surtout pas devenir une arche de Noé. Pourvu qu'il ne s'agisse pas d'un " procréateur en puissance » (p. 103)! Quand Nagg réclame sa bouillie, Hamm l'insulte violemment: «Maudit progéniteur!»(p. 21). Au terme attendu de «procréateur», Beckett substitue celui de «progéniteur». Toute génération, toute reproduction est impitoyablement rejetée. La relation père-fils est conflictuelle :

« Hamm - Salopard! Pourquoi m'as-tu fait?

Nagg - Je ne pouvais pas savoir.

Hamm - Quoi ? Qu'est-ce que tu ne pouvais pas savoir?

Nagg - Que ce serait toi. » (p. 67)

Hamm souhaite un monde sans «progéniteur ». La reproduction nous inscrit dans l'illusion d'un temps cyclique, renouvelable, dans l'espoir d'un éternel retour. Beckett ne cesse de railler cet espoir de reproduction entretenu par le rite, le mythe ou la religion.

On peut être étonné de la petite place réservée aux femmes dans cette condamnation de la reproduction. Clov qui plie ses draps soigneusement n'est qu'une inversion, une femme de comédie. Nell n'est plus qu'une marionnette ridicule, sans " guibolles ". C'est l'homme, le mâle, le responsable, le créateur des mythes et de rites, le procréateur occasionnel parce qu'il possède les graines, sperma. La terre-mère n'est qu'un réceptacle. Déméter, pas plus que Nell, n'est accusée. Nagg raconte ainsi à Hamm son enfance sans mère :

«Nagg - Qui appelais-tu quand tu étais tout petit et avais peur, dans la nuit? Ta mère? Non. Moi. On te laissait crier. [...] J'espère que le jour viendra où tu auras vraiment besoin que je t'écoute, et besoin d'entendre ma voix, une voix. (Un temps.) Oui, j'espère que je vivrai jusque-là, pour t'entendre m'appeler comme lorsque tu étais tout petit, et avais peur dans la nuit, que j'étais ton seul espoir. » (p. 75)

Curieux monde où les pères mettent au monde des fils pour les laisser crier de peur ${ }^{(34)}$. L'exemple vient d'en haut, de Dieu le Père lui-même, qui a laissé son fils crier sur la Croix. La Passion est le récit scandaleux d'un homme qui souffre par la faute de son père. Hamm a beau nier l'existence de ce Père, "Le salaud! Il n'existe pas !" (p. 74), crucifié sur son fauteuil, à la fin de la pièce, il souffre comme le Christ, et réclamera en vain l'intervention salvatrice.

Le seul être féminin de la pièce qui pourrait se reproduire est une puce ! Cela donne lieu encore à une pantalonnade comique. Clov sent la bête dans l'entrejambes de son pantalon. Suit alors une scène burlesque qu' on pourrait trouver chez Chaplin ou Keaton: Clov verse «frénétiquement» (p.49) de l'insecticide dans son pantalon pour

(34) Beckett écrit sa pièce après la mort de son père. 
qu'elle se tienne « coïte». Hamm rectifie : « coite», pas « coïte». Mais il ne peut s'empêcher de faire ce mauvais jeu de mots : «Mais voyons! Si elle se tenait coïte nous serions baisés. » (p. 49)

Se faire « baiser », c'est entrer à nouveau dans le processus infernal de la reproduction, accepter à nouveau que les pères et les fils se déchirent. Et pourtant il est difficile d'abandonner tout espoir. Hamm rêve encore de «baiser» :

« Mais taisez-vous, taisez-vous, vous m'empêchez de dormir. (Un temps.) Parlez plus bas. (Un temps.) Si je dormais je ferais peut-être l'amour. J'irais dans les bois. Je verrais... le ciel, la terre, je courrais. On me poursuivrait. Je m'enfuirais. (Un temps). Nature!» (p. 31)

Hamm affame ses parents dans leurs poubelles, réclame un boueux pour les éliminer et tyrannise Clov, son fils adoptif, dont il attend vainement la pitié. Mais le fils tyrannisé ne réussit pas à briser le lien qui l'attache à son père. Il ne franchit jamais le « seuil ». A quoi sert donc à Hamm d'avoir un fils si inutile ? Hamm répond à cette question fort drôlement. Il sert à lui donner « la réplique» (p.78). C'est sa manière de reconnaître sa dette envers ce personnage qu'il n'estime guère. Mais ce fils adoptif est aussi, bien qu'il s'en défende, sa « réplique », une image de lui-même qui montre combien il est difficile d'en finir avec toute reproduction.

\section{L'impossible tas}

Nous avons vu tout au long de cette étude qu'il est difficile, pour les personnages, de passer le seuil pourtant espéré et de se résoudre à définitivement « finir de perdre ». Il est douloureux, pour Hamm, de laisser à la porte tout espoir de retour, de résurrection ou de reproduction, d'espérer encore apercevoir « une voile », « une nageoire » ou « une fumée » (p. 45). Il doit lutter contre l'idée séduisante que tout puisse encore recommencer. Il est tellement humain de vouloir espérer la fin de la dégradation physique ou de souhaiter un monde moins cruel. Déjà aveugle et paralysé, presque abandonné par son fils adoptif, Hamm sait bien que l'espoir n'est qu'un leurre. Que peut-il encore attendre ? Le passage enfin vers un ailleurs, la sortie de ces limbes où il est confiné, la fin « inouïe » (p. 65), le moment où la frontière sera enfin franchie, et l'agrégation finale réalisée. N'est-ce pas le dernier espoir dont il faut se déprendre, l'ultime illusion?

Les élèves sont-ils sensibles à cette méditation sur le temps humain que propose la pièce ? Les rites de passage qu'ils ont découverts à l'occasion de ce travail sur l'œuvre de Beckett fabriquent un temps cohérent et ordonné bien éloigné de l'expérience commune que nous avons du temps. Le temps vécu au quotidien est heurté, saccadé, hésitant, comme le marquent toutes les pauses qui émaillent le texte. Pourquoi ne pas se pencher alors sur le roman que compose Hamm ? Pourquoi écrit-il ? Peut-être pour raccommoder le temps, le tisser? Il est conscient de son incapacité à terminer son histoire, et à sortir du temps morcelé qui est le sien. La suite des passés simples qui structurent son récit souligne, jusqu'à la parodie, l'illusion que tout s'enchaîne logiquement, post hoc propter hoc, mais en réalité le texte ne progresse pas. Le linceul de Pénélope n'est jamais fini. Le piétinement et l'enlisement des imparfaits ainsi que les digressions montrent la difficulté à sortir de cette marge littéraire pour accéder enfin à l'agrégation finale, à « l'impossible tas ». Il écrit : «Il faisait ce jour-là, je m'en souviens, un froid extraordinairement vif...» (p. 69) mais plus loin, on lit : «Il faisait ce jour-là, je me rappelle, un soleil vraiment splendide... » (p. 69). Et plus loin encore : «Il faisait ce jour-là, je m'en souviens, un vent cinglant...» (p. 70). Le temps ne passe pas, le temps hésite. Comment organiser alors un récit miné par d'incessantes remarques la plupart du temps 
contradictoires? Hamm, créateur minable, ne peut terminer ce roman qui ne sera pas mieux réussi que le pantalon de l'Anglais ou le monde divin. L'acte créateur, sans cesse moqué, est voué à l'échec. Tout ce qui donnerait l'illusion d'un monde ordonné et d'une temporalité cohérente est abandonné, comme livré à une dérive onirique qui bannirait étrangement tout ce qui structure et organise : le sexe, la filiation et les lois du récit. L'histoire imaginée par Hamm prolifère ou bourgeonne, de manière anarchique.

« Hamm - Je n'en ai plus pour longtemps avec cette histoire. (Un temps.) A moins d'introduire d'autres personnages. » (pp. 72-73)

Mais Hamm sait bien que ces rajouts ultimes seraient encore pires. Il a beau avouer sa passion de l'ordre, rien n'y fait :

« J'aime l'ordre. C'est mon rêve. Un monde où tout serait silencieux et immobile et chaque chose à sa place dernière, sous la dernière poussière. » (p. 76).

Un monde où tout est « silencieux et immobile et chaque chose à sa place dernière » est un monde mort. Un monde de cendres. Dès le début de la pièce Hamm souhaite que le temps s'accélère enfin pour hâter sa mort. « Ça ne va pas vite. » regrette-t-il (p. 24). Il s'inquiète souvent : "C'est une fin de journée comme les autres, n'est-ce pas Clov?» (p. 26) Et Clov essaie de le rassurer : "Quelque chose suit son cours. » (p. 26) Il faut se persuader sans cesse que, malgré les apparences, «ça avance. » (p. 27). Hamm espère que la Nature le conduira vers la fin :

« Mais nous respirons, nous changeons! Nous perdons nos cheveux, nos dents ! Notre fraîcheur! Nos idéaux!» (p. 23)

Le passage attendu est celui de la fin du temps, de la fin des temps. Fin de partie est une méditation eschatologique sur le moment où retentira enfin la trompette dernière, trompette représentée dérisoirement par le réveil de Clov (p. 65).

«Les grains s'ajoutent aux grains, un à un, et un jour, soudain, c'est un tas, un petit tas, l'impossible tas. » (p. 14)

C'est ce « soudain » qui est important. C'est le moment rapide du passage alors qu' on vit dans un continuum maussade. Les élèves sont intrigués, à juste titre, par cette image répétée du tas de grains qui lie le début à la fin de la pièce :

«Instants sur instants, plouff, plouff, plouff, comme les grains de mil de... (il cherche)... ce vieux Grec, et toute une vie on attend que ça fasse une vie. (Un temps. Il veut reprendre, y renonce. Un temps.) Ah, y être! (Il siffle. Entre Clov, le réveil à la main. Il s'arrête à côté du fauteuil.) Tiens ! Ni loin ni mort? » (p. 91)

Comment expliquer que, "soudain », une suite d'instants insignifiants fasse « une vie »? Le réveil de Clov ne peut qu'indiquer un temps cyclique. Comment expliquer que " soudain » les grains fassent « un tas » et que se termine enfin l'infernale répétition ? Le passage magique de ce « seuil», où la somme est plus que l'addition des parties, mobilise toute l'attention de Hamm. Le «vieux Grec», Eubulide de Milet parait-il, est l'auteur de ce paradoxe antique célèbre, le paradoxe du tas, dit paradoxe " sorite ${ }^{(35)}$. Quand donc les grains finissent-ils par faire un tas ? Comment rendre compte

(35) Le sorite, vient du mot grecsoros : le tas. Quand commence un tas de blé ? Un grain n'est pas un tas, ajouter un grain à un grain ne fait pas un tas, ni en ajouter deux, ni trois, quand donc ? Diogène Laërce attribue ce paradoxe à Eubulide de Milet ( IV $^{\mathrm{e}}$ siècle avant J.C.). 
rationnellement de ce passage soudain de la quantité à la qualité ? Hamm sent bien que le tout, « le tas », « la vie » n'est pas seulement la somme des parties. Fin de partie, c'est la fin des parties et le passage espéré à la totalité signifiante. La dernière illusion?

Mais la maîtrise de ce « passage » échappe totalement à Clov, comme à Hamm. L'aide rituelle semble bien pauvre. Que reste-t-il alors à faire? A attendre, à éprouver le temps dans ce qu'il a de plus pesant. Tous attendent. Clov est, lui aussi, à la fin de la pièce, sur le départ, en attente, figé sur le seuil, en costume de voyage. Et le «père », sans le soutien de son " fils », doit lui aussi attendre son passage sur son fauteuil roulant. Et que faire, en attendant, comme dans la pièce précédente, En attendant Godot, sinon répéter des gestes qu'il est bien difficile de qualifier maintenant de rituels puisqu'ils sont vidés d'espoir et de sens. Les rites ne sont-ils pas destinés, comme le remarquait Pierre Bourdieu, à « donner au moins l'apparence d'un sens, d'une raison d'être, à ces êtres sans raison d'être que sont les êtres humains, de leur donner le sentiment d'avoir une fonction ou, tout simplement, une importance, de l'importance, et de les arracher ainsi à l'insignifiance ${ }^{(36)}$ ? Et que vaut un rituel sans l'adhésion de l'initié et la foi de l'initiateur? Le spectateur est amené, dans la tirade finale, à éprouver ce temps qui n'en finit pas, à partager avec Hamm cette attente fébrile et hypothétique.

\section{Une passion}

Le théâtre, comme jeu et répétition, comme exemplum, est l'ultime rite. Hamm se résout donc, à la fin de la pièce, à jouer la comédie. Mais il est seul maintenant, sans personne pour lui donner la réplique, clown sanglant et solitaire. La scène qu'il interprète ne peut être que parodique, puisque la parodie est finalement l'aveu d'une impuissance. La fin de la pièce parodie son propre début: Hamm imite le jeu des tissus de Clov. Mais elle parodie aussi le texte sacré de la Passion du Christ, l'histoire d'un autre passage célèbre, d'une « Pâque », où le fils dut seul affronter la mort.

«Puisque ça se joue comme ça... (il déplie son mouchoir)... jouons ça comme ça... » (p. 110)

Hamm va jouer, lui aussi, à se dépouiller avant de mourir. Il abandonne tout ce qui peut le retenir. Il renonce à la gaffe qui faisait de lui un nocher infernal, il jette le sifflet qui le liait à Clov, il se sépare de son chien qu'il serrait contre lui comme le fait un enfant. Il ne garde que ce mouchoir, ce «vieux linge » familier que ne lui tend aucune Véronique. Il le déplie et l'interpose entre lui et le public, attendant que le rideau tombe vraiment. La pièce se termine ainsi, étrangement. Comme si Beckett avait voulu nous entraîner jusqu'à ce seuil, devant cet écran sanglant et énigmatique, jusqu'à ce moment où l'on attend la fin.

Les élèves, habitués à des œuvres closes, structurées, dont la morale est évidente, n'aiment guère, en général, rester ainsi sur leur « fin ». Mais ils sentent confusément que ce qui les saisit dans le texte de Beckett, c'est justement ce qui contrarie leur désir de comprendre. Comme Hamm, ils attendent que tout ce texte fasse « une vie », constitue enfin un « tas » signifiant. Cette difficulté d'accéder au sens en fascine beaucoup.

Pour finir l'étude, pourquoi ne pas lire, à haute voix, en classe, le dernier poème de Beckett, son dernier monologue, intitulé Comment dire, écrit en 1988, peu avant sa mort dans la solitude d'une maison de retraite? C'est le dernier mouchoir ensanglanté qu'il nous tend, l'ultime écran où il répète sa difficulté à dire l'indicible de l'attente :

(36) Pierre Bourdieu, «Les rites comme actes d'institution », in Actes de la recherche en sciences sociales, Vol. 43, juin 1982. p. 58. 
«[...] folie que de vouloir croire entrevoir quoi -

quoi -

comment dire -

et où -

que de vouloir croire entrevoir quoi où -

où -

comment dire -

là -

là-bas -

loin -

loin là là-bas -

à peine -

loin là là-bas à peine quoi -

quoi -

comment dire -

vu tout ceci -

tout ce ceci-ci -

folie que de voir quoi -

entrevoir -

croire entrevoir -

vouloir croire entrevoir -

loin là là-bas à peine quoi -

folie que d'y vouloir croire entrevoir quoi -

quoi -

comment dire -

comment dire " ${ }^{(37)}$

(37) «A l'inverse de tant d'auteurs qui, en vieillissant, engagent des projets voués par la mort à l'anéantissement, contraint et forcé, Beckett, achève lui-même l'extinction de son entreprise. Cette extinction est, de plus, d'une certaine manière, en germe dès l'un de ses premiers textes. Dans son essai sur Proust, en 1930, Beckett écrit: "La pulsion artistique ne va pas dans le sens d'une expansion mais d'une contraction. L'art est l'apothéose de la solitude” (in Proust, Éditions de Minuit, 1990, p. 75). En sa concentration l'œuvre terminale propose sa colorature sombre, sa matière subtile et diaphane. Elle permet pour le créateur de signifier tout un poids de ténèbres, tout l'ombre de l'ombre comme dans Comment dire, le dernier texte de Beckett écrit quelques semaines avant sa mort [...] Juste avant la mort, presque dedans, ce poème annonce une dernière expérience des limites. Là alors, la seule et paradoxale image portée au noir. Celle que l'auteur, entouré de vieilles dames, muet et immobile, fixant le poste de télévision, regarde une dernière fois avant de mourir. La "seule" image qui fait écho à celle évoquée en cet aveu capital de 1948 écrit dans une lettre inédite à Jacques Kober: "Je ne connais que la vieille image nä̈ve et sourde qui n'ajoute rien, n'élargit rien, ne fait que renvoyer à l'affolement dont elle sort, comme le cri absurde à la douleur et à la joie". " J.-Paul Gavard-Perret (http://www.espritsnomades.com/sitelitterature/beckett/beckett.html) 